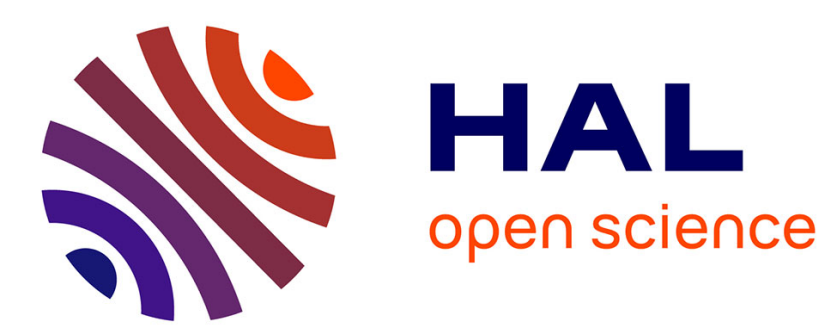

\title{
Compromis institutionnels et hybridations entre recherche publique et recherche privée
}

Maurice Cassier

\section{To cite this version:}

Maurice Cassier. Compromis institutionnels et hybridations entre recherche publique et recherche privée. Revue d'économie industrielle , 1997, 79, pp.191-212. halshs-01894060

\section{HAL Id: halshs-01894060 \\ https://shs.hal.science/halshs-01894060}

Submitted on 12 Oct 2018

HAL is a multi-disciplinary open access archive for the deposit and dissemination of scientific research documents, whether they are published or not. The documents may come from teaching and research institutions in France or abroad, or from public or private research centers.
L'archive ouverte pluridisciplinaire HAL, est destinée au dépôt et à la diffusion de documents scientifiques de niveau recherche, publiés ou non, émanant des établissements d'enseignement et de recherche français ou étrangers, des laboratoires publics ou privés. 


\title{
Persée \\ http://www.persee.fr
}

\section{Compromis institutionnels et hybridations entre recherche publique et recherche privée}

\author{
Maurice Cassier \\ Cassier Maurice, . Compromis institutionnels et hybridations entre recherche publique et recherche privée. In: Revue \\ d'économie industrielle. Vol. 79. 1er trimestre 1997. L'économie industrielle de la science. pp. 191-212.
}

Voir l'article en ligne

Tandis que des travaux en économie de la science insistent sur la précarité des contrats de recherche entre l'université et l'industrie, en raison des asymétries d'information auxquelles ils sont soumis et de l'incompatibilité des systèmes d'incitation des deux types d'institutions, nous démontrons la possibilité d'établir des formules de compromis entre bien privé, bien collectif et bien public. Ce régime d'appropriation modérée, qui fait qu'une entreprise ne capte pas l'ensemble des résultats du contrat qu'elle finance, favorise le développement des stocks de connaissances et la croissance des externalités de la recherche. Plus généralement, l'organisation linéaire de la science et de la technologie, confinées dans des institutions séparées, cède le pas à des interactions récurrentes entre université et industrie, ainsi qu'à des intégrations verticales dans les différentes institutions. Dans ce contexte, les universités sont amenées à apprendre à utiliser les brevets comme des outils de réservation de leurs inventions et des outils de coordination avec les firmes.

\section{Avertissement}

L'éditeur du site «PERSEE »- le Ministère de la jeunesse, de l'éducation nationale et de la recherche, Direction de l'enseignement supérieur, Sous-direction des bibliothèques et de la documentation - détient la propriété intellectuelle et les droits d'exploitation. A ce titre il est titulaire des droits d'auteur et du droit sui generis du producteur de bases de données sur ce site conformément à la loi n`98-536 du 1er juillet 1998 relative aux bases de données.

Les oeuvres reproduites sur le site «PERSEE » sont protégées par les dispositions générales du Code de la propriété intellectuelle.

Droits et devoirs des utilisateurs

Pour un usage strictement privé, la simple reproduction du contenu de ce site est libre.

Pour un usage scientifique ou pédagogique, à des fins de recherches, d'enseignement ou de communication excluant toute exploitation commerciale, la reproduction et la communication au public du contenu de ce site sont autorisées, sous réserve que celles-ci servent d'illustration, ne soient pas substantielles et ne soient pas expressément limitées (plans ou photographies). La mention Le Ministère de la jeunesse, de l'éducation nationale et de la recherche, Direction de l'enseignement supérieur, Sous-direction des bibliothèques et de la documentation sur chaque reproduction tirée du site est obligatoire ainsi que le nom de la revue et- lorsqu'ils sont indiqués - le nom de l'auteur et la référence du document reproduit.

Toute autre reproduction ou communication au public, intégrale ou substantielle du contenu de ce site, par quelque procédé que ce soit, de l'éditeur original de l'oeuvre, de l'auteur et de ses ayants droit.

La reproduction et l'exploitation des photographies et des plans, y compris à des fins commerciales, doivent être autorisés par l'éditeur du site, Le Ministère de la jeunesse, de l'éducation nationale et de la recherche, Direction de l'enseignement supérieur, Sous-direction des bibliothèques et de la documentation (voir http://www.sup.adc.education.fr/bib/ ). La source et les crédits devront toujours être mentionnés. 


\section{COMPROMIS INSTITUTIONNELS ET HYBRIDATIONS ENTRE RECHERCHE PUBLIQUE ET RECHERCHE PRIVÉE}

Mots-clés : Université, industrie, contrats, bien public, bien privé.

Key words : University, industry, research contracts, public good, private good.

\section{INTRODUCTION}

L'enquête de Cohen, Florida and Goe (1994) sur les centres de recherche université/industrie aux États-Unis, montre que les industriels participants sont susceptibles d'imposer des restrictions à la libre circulation des résultats académiques (1). Elle fait écho aux craintes exprimées par Dasgupta et David (1994) quant à la fragmentation des réseaux d'échanges académiques et à l'affaiblissement du système de la " science ouverte " provoqués par la prolifération des collaborations entre l'université et l'industrie et l'adoption par l'université des normes de la science privée. La conclusion de l'étude est toutefois nuancée : 1- ces restrictions sont balancées par les bénéfices des avancées technologiques, au moins à court terme, 2- ces centres ont renforcé les canaux par lesquels la connaissance émanant de l'université est transférée dans l'industrie, et, dans l'autre sens, l'information sur les besoins de l'industrie vient stimuler la recherche universitaire. Autrement dit, les collaborations avec l'industrie ne se réduisent pas à un prélèvement des stocks de recherche de l'université. Elles donnent également lieu à des apports de ressources pour celle-ci. Par exemple, $44,8 \%$ des centres indiquent qu'ils ont accès aux données et installations de l'industrie, 3- le coût social de ces restrictions est sans doute

(1) Les chercheurs appartenant à de tels centres sont moins enclins à partager leur information, avec leurs collègues des centres de recherche université/industrie $(13.4 \%)$, de l'université $(21,3 \%)$, des autres universités $(28,6 \%)$, avec le public $(41.5 \%)$. Près de $35 \%$ des centres indiquent que les entreprises sont susceptibles de demander la suppression d'informations dans les papiers qui leur sont soumis et $52.5 \%$ qu'elles peuvent imposer un délai de publication. Les auteurs soulignent toutefois : "Note that our surve y asked UIRC directors to report whether participating companies can delay publication or require that information be deleted. Our data do not indicate the actual frequencr with which publication is delaved or information deleted. Furthermore, our surver data do not include what kind of information (e.g., proprietary) companies can ask to hare deleted". 
moindre si l'alternative consiste à réaliser la même recherche dans l'industrie, avec des restrictions plus fortes encore, 4 - il s'avère que la productivité académique de ces centres, mesurée par le nombre de papiers de recherche par million de dollars dépensé, est comparable à celle de l'université dans son ensemble.

Est-il possible de construire des arrangements contractuels qui favorisent la coopération entre des acteurs dont les rationalités sont a priori totalement divergentes ? Pour Dasgupta et David, 1994, ce type de mariage entre la culture de la science ouverte et celle de la science privée est fondamentalement précaire et menace la libre circulation des connaissances.

Nous montrerons que les acteurs parviennent à mettre au point des solutions de compromis qui organisent un double flux de résultats, vers les réseaux académiques, d'une part, vers les cercles privés de la R\&D des firmes, d'autre part. Si des tensions sur la privatisation de certaines connaissances existent, les institutions scientifiques sont à même d'apprendre à gérer leur stock de recherche, par exemple en accordant des exclusivités sur des applications spécifiques tout en maintenant les connaissances générales qu'elles détiennent dans le domaine public. Elles peuvent encore adopter des mesures qui stimulent la divulgation rapide de leurs résultats. Les chercheurs utilisent couramment plusieurs degrés de confidentialité et d'accessibilité des connaissances, depuis les données propres au laboratoire, éventuellement très confidentielles, les données échangées dans le cercle de leurs partenaires scientifiques, les données versées dans l'espace public et accessibles à tous les utilisateurs potentiels. Ils sont capables d'inventer de nouvelles solutions en terme de droit d'accès aux données, à l'instar des guidelines élaborées par les chercheurs des programmes européens de biotechnologie.

L'élaboration de solutions de compromis qui permettent de satisfaire les objectifs mutuels des deux parties et de distribuer des résultats aussi bien dans la sphère privative que dans l'espace public est d'autant plus cruciale que les coopérations entre l'université et l'industrie participent à une nouvelle organisation de la recherche. Dans un tel schéma, iI s'agit moins de régler un problème ponctuel de transfert de connaissances et de technologies, que de construire des coopérations durables pour stimuler les Pasteurs-like activities, pour reprendre l'expression de Nelson et Romer (1994). Il s'agit également de gérer les situations hybrides auxquelles sont confrontées les laboratoires publics qui produisent des connaissances et des techniques qui ont une double valeur d'usage, académique et industrielle. L'intégration des laboratoires dans l'économie ne signifie pas nécessairement un glissement d'un ordre public vers un ordre privé, mais plutôt un apprentissage pour maitriser les rapprochements et les échanges entre les deux secteurs. Gluck et al., 1987, qui ont enquêté sur les collaborations université/industrie dans les sciences de la vie, recommandent la formation des chercheurs à la gestion de ces relations. Ils observent en effet que les universitaires les moins expérimentés en la matière peuvent restreindre leurs communications avec leurs collègues au-delà de ce qui est nécessaire. 
Nous fonderons notre démonstration sur l'étude de 158 contrats de recherche conclus sur la période 1972-1991, entre 94 entreprises et 3 centres de biotechnologie à Compiègne, Toulouse et Marseille. Ces accords sont le plus souvent, pour $92 \%$ d'entre eux, des contrats bilatéraux par l'intermédiaire desquels les firmes acquièrent individuellement des connaissances et des technologies auprès de l'université. C'est une forme de contrat qui facilite la préservation de la confidentialité et de l'exclusivité des résultats. Les contrats multilatéraux ne sont toutefois pas absents, soit sous forme de coopération triangulaire, entre un laboratoire concepteur d'un instrument scientifique, un industriel fabricant et un utilisateur, soit sous forme de réseaux coopératifs plus étendus. Ce travail a mobilisé les documents écrits des accords ainsi que les interprétations des partenaires engagés souvent depuis le début des années 1970 dans de telles collaborations. Cette histoire est une ressource, pour les acteurs, qui ont progressivement mis au point des outils contractuels, et pour l'analyste, qui peut observer des « réseaux technico-économiques » (Callon, 1992) en train de s’étendre.

La première section traite de l'élaboration des outils contractuels utilisés pour réguler l'attribution des résultats entre les parties. La seconde section décrit les types d'arrangements observés et analyse leur impact sur la création et la circulation des connaissances et des technologies. La troisième section envisage l'émergence d'institutions hybrides entre la science et le marché, entre le domaine public et le domaine privé de la recherche.

\section{1. - ÉLABORER DES OUTILS CONTRACTUELS POUR RÉGULER L'APPROPRIATION DES PRODUITS DE LA RECHERCHE}

\subsection{Ecrire un contrat pour réguler le problème de l'attribution des résultats}

C'est pour une large part l'attribution et l'appropriation des résultats qui justifient l'écriture d'un contrat de recherche (2). Lors d'une collaboration établie entre l'INSA de Toulouse et une société de biologie moléculaire, commencée sans contrat explicite, les deux parties ont éprouvé la nécessité de rédiger une convention dès lors que les résultats obtenus pouvaient avoir une valeur d'usage industrielle. L'absence de contrat écrit peut rendre la coopération instable, d'autant que selon Mousseron, 1995, juriste spécialiste de la propriété intellectuelle, "Le régime juridique des contrats d'entreprise n'est pratiquement pas fixé par la loi. En découle l'importance de la rédaction des conventions lorsqu'elles portent sur des informations non appropriées -non brevetées-, car on ne se fonde que sur la théorie générale des contrats. Il faut donc prévoir avec grand soin les obligations de chaque partie et les conséquences de la fin du secret sur le contrat...».

(2) «Un mécanisme de partage du surplus créé doit en conséquence faire partie intégrante de l'accord et détermine en grande partie l'efficacité même de la coopération " (Brousseau. 1993). 
La rédaction d'un accord sur la propriété, même non signé par les services de propriété industrielle des deux parties, comme ce fut le cas pour les guidelines des consortia européens en biotechnologie, est un élément de stabilité de la collaboration : 1- il constitue une mémoire tangible qui pourrait être sollicitée en cas de litige pour rappeler le sens des engagements initiaux. A ce titre, c'est un outil de prévention des disputes et de dissuasion des conduites opportunistes, qui peuvent être aiguisées en fin de contrat, 2- dans la mesure où il a été négocié et écrit de manière concertée, il est devenu le bien commun des contractants, ce qui facilite la convergence des interprétations (soit une économie de coordination), 3- son inscription dans des documents facilite la circulation et la standardisation des arrangements d'une université à l'autre, 4- il assure l'ancrage d'un accord singulier dans le système institutionnel et juridique. En dernier ressort, l'exécution des clauses de l'accord est garantie par l'autorité judiciaire qui est généralement explicitement mentionnée dans la convention (3).

La rédaction de ces clauses requiert la participation conjointe des chercheurs des laboratoires et des gestionnaires des universités. Nul autre que le chercheur, parfaitement au fait de l'état des connaissances sur le sujet, ne peut déterminer les antériorités qu'il détient vis à vis de ses partenaires ou les exclusivités qu'il peut leur accorder sans compromettre le développement de ses recherches. C'est également lui le mieux placé pour négocier les délais de publication à consentir à l'industriel compte tenu de la concurrence qu'il fait à ses homologues universitaires. Il doit toutefois recourir à des conseils juridiques, le plus souvent externes, qui sont au fait des «bonnes pratiques » en vigueur dans l'écriture des contrats et des brevets. L'assistance de conseils en brevets ou l'intégration de juristes dans les services des universités permet de réduire l'asymétrie de compétences dont elles souffrent vis à vis des firmes en matière de propriété industrielle. Il s'agit également d'organiser la sélection et la circulation des bonnes pratiques contractuelles au sein des réseaux d'universités à l'instar du réseau CURIE ou du GIE Ressources qui regroupent les grandes associations de recherche sous contrat françaises.

\subsection{Les problèmes spécifiques d'établissement et de gestion de la propriété dans les contrats de recherche}

Les contrats représentent une modalité particulière d'appropriation des connaissances et des technologies, complémentaire du droit de la propriété

(3) L'écriture de règles d'attribution des résultats des recherches ne suffit pas à faire tenir le lien contractuel. Tout d'abord. il existe des collaborations sans contrat écrit. Il s'agit le plus souvent de la fourniture par l'entreprise de matériel biologique privé en échange des résultats scientifiques obtenus par les chercheurs. L’industriel bénéficie d'un droit d'accès prioritaire aux connaissances et de la propriété des retombées industrielles. L'accord est fondé sur la confiance et la réciprocité des échanges entre les partenaires. Ensuite. quand bien même le contrat est-il écrit. la confiance facilite grandement son exécution. Les coûts de surveillance sont réduits et le crédit mutuel que s"accordent les partenaires aide grandement à la résolution des conflits qui peuvent survenir. Enfin, la connaissance des conventions et des règles d'action de son partenaire favorise la stabilité de l'accord (Jacquemin. 1987). 
industrielle. Les techniques contractuelles, par le jeu des obligations de communiquer à un bénéficiaire exclusif et de ne pas communiquer à des tiers, créent un droit de réservation sur des résultats très hétérogènes, brevetables ou non brevetables (Reboul, 1978). Le bénéficiaire peut utiliser son droit de réservation temporaire sur les produits du contrat pour évaluer leur valeur d'usage industrielle et commerciale, généralement indéterminée au moment de l'accord, et le cas échéant décider de les breveter. Les contrats de recherche livrent de nombreux résultats intermédiaires qui seront éventuellement réutilisés par la recherche industrielle pour produire des innovations. Il s'agit par exemple de matériels de recherche qui ne sont pas disponibles sur le marché et qui ne sont pas couverts par des brevets. Ainsi les chercheurs ont-ils mis au point des lettres-contrat pour assurer la circulation de matériels de laboratoire très spécifiques, seulement disponibles auprès de la source qui les a isolés ou construits.

Les contrats de recherche doivent réguler des problèmes particuliers pour l'appropriation de leurs produits.

Premièrement, au moment de l'accord, les contractants détiennent une information incomplète sur les résultats, (sur leur obtention, leur forme, et sur leur valeur d'usage). Le contrat, par nature incomplet, s'inscrit dans un processus temporel, et fixe des procédures de qualification des résultats, au fur et à mesure de leur obtention et de leur évaluation. Si l'accord prescrit une répartition ex ante de la propriété (propriété unique, partagée, ou séparée), il prévoit des négociations en cours d'exécution pour décider de la forme et du statut économique et juridique des résultats (dossier technique secret, brevet, licence, publication, etc.). Cela peut être la tâche d'un comité de coordination qui réunit des membres désignés par les contractants pour les représenter. Ce comité exerce une surveillance mutuelle sur le déroulement de la recherche, assure l'échange des informations entre les deux parties, a un rôle d'arbitrage pour décider de l'orientation des travaux et de la qualification des résultats. Pour Pisano, 1991, ces négociations futures ne font qu'ajouter de l'incertitude à l'exécution des contrats de recherche et peuvent être sources de coûteuses disputes, dues à l'opportunisme ou à d'honnêtes différences d'opinion. On peut rétorquer que l'incomplétude des contrats est parfaitement adaptée au caractère incertain de l'activité de recherche. Les acteurs disposent d'une rationalité non seulement limitée, mais qui se limite à l'épreuve des faits (Livet et Thévenot, 1991), et qui ajuste ses décisions dans l'action, au fur et à mesure des informations qu'elle engrange.

Deuxièmement, l'exécution des contrats de recherche est menacée par des difficultés qui dérivent des asymétries d'information entre les acteurs (Arrow, 1962, Williamson, 1981, Dasgupta et David, 1994). Première difficulté, l'inégale dotation d'informations des acteurs sur les objets échangés peut attiser leur opportunisme. Le laboratoire pourrait être tenté de dissimuler certains résultats à son partenaire, qui ne peut les observer et les identifier parfaitement. A cela, plusieurs réponses possibles : a- le bénéficiaire est protégé par le droit commun ou par le droit contractuel qui déterminent pour le prestataire de 
recherche une obligation de communication de l'ensemble des résultats obtenus (Reboul, 1978), b- la convention peut fixer un droit de visite qui facilite le suivi et la surveillance des travaux, c- à la différence de la relation d'agence qui existe par exemple entre un avocat et son client (Perrot, 1991), la firme contractante et le laboratoire disposent de compétences et d'informations assez largement équivalentes. Près de $90 \%$ des demandeurs de recherche de notre population possèdent une expertise interne en matière de recherche-développement (les responsables des laboratoires industriels sont fréquemment d'anciens universitaires) et, lorsqu'ils n'en sont pas équipés, ils recourent à l'expertise d'un tiers, par exemple un conseiller technique régional à la disposition des PME. Seconde difficulté, les asymétries d'information posent de redoutables problèmes de convergence des interprétations des contractants sur les objets produits et les évènements qui surviennent, dès lors que l'accord n'est plus fondé sur « l'évidence d'un contexte commun " (Livet et Thévenot, 1991). On relève ainsi des mésententes persistantes entre universitaires et industriels sur la valeur d'usage industrielle d'un prototype de laboratoire, que les uns estiment dans un milieu modèle de laboratoire, et que les autres jugent dans un milieu industriel. Ces difficultés de qualification commune des objets et des faits peuvent trouver une solution dans un apprentissage mutuel qui se produit au cours de collaborations de longue durée ou de contrats répétés.

Troisièmement, les contrats de recherche doivent régler le problème de la protection, de la transmission et de l'appropriation des connaissances tacites, dans la mesure où il est coûteux sinon impossible de les spécifier dans un contrat (Dasgupta et David, 1994). Or la valeur d'usage d'une connaissance codifiée ou d'un prototype transféré par contrat dépend souvent des connaissances tacites qui leur sont associées. L'accord peut tout d'abord prescrire une obligation d'assistance technique au bénéfice du demandeur de recherche en cas de dépôt de brevet ou de concession de licence. Il s'efforce ensuite de fixer les modalités de la transmission de ces connaissances dans le cadre d'échanges interpersonnels. Sont ainsi programmées des réunions, des visites sur les sites scientifiques et industriels, des démonstrations en laboratoire industriel ou en usine, des formations. Au-delà de la précision des obligations contractuelles, le laboratoire est incité à communiquer suffisamment de connaissances tacites à son partenaire s'il bénéficie de retours sous forme de royalties ou s'il veut entretenir avec lui une coopération durable.

Quatrièmement, il apparaît des problèmes particuliers pour la répartition des résultats des recherches conjointes, dès lors qu'ils résultent de l'association ou de la combinaison des actifs et des travaux des deux parties (Gaffard, 1989). Les partenaires devront consentir un coût de négociation ex post pour évaluer une redevance, établir une copropriété ou encore pour délimiter les champs respectifs attribués à chaque partie dans le cas d'une propriété séparée. Par exemple, lors de la concession d'une licence de savoir-faire portant sur un prototype de dosage enzymatique, l'attribution d'une redevance au laboratoire fut justifiée par l'évaluation rétrospective de l'ensemble des investissements consentis pour le mettre au point. Les laboratoires doivent se doter de l'ins- 
trumentation nécessaire pour évaluer leur stock de recherche et faire appel à l'expertise, de plus en plus étoffée, des services gestionnaires de la recherche des universités pour négocier cette répartition avec les entreprises.

Cinquièmement, dans la mesure où le laboratoire détient un actif localisé, et parfois unique, dont l'entreprise a un fort besoin pour développer une innovation, il peut utiliser cette asymétrie pour renégocier un contrat dans des conditions très favorables qui lui permettent d'exiger une part accrue de la rente d'innovation (Pisano, 1991). Prenons un exemple : le laboratoire de lipolyse enzymatique de Marseille, inventeur d'un instrument scientifique, s'est allié avec un laboratoire de l'université de Toulon pour la réalisation de la partie optique et logicielle de l'appareil. Lors du transfert de l'innovation à une petite société d'instrumentation créée pour l'occasion, le laboratoire de Toulon, propriétaire d'un brevet sur la partie logicielle, refuse d'en communiquer les sources à l'entreprise. La nouvelle société se trouve dans une situation de dépendance totale puisqu'elle doit passer par l'université de Toulon pour effectuer toute modification ou adaptation. Elle fut contrainte de redévelopper entièrement la partie logicielle de l'instrument. Dans un autre cas, symétrique, le laboratoire de Compiègne, détenteur de connaissances et de savoirs-faire spécifiques et encore peu explicités, a pu contrer les vélléités opportunistes d'une firme qui entendait s'approprier les bénéfices d'un prototype inventé à l'université. La simple menace des universitaires de ne plus lui communiquer d'informations a suffit à faire taire son opportunisme.

Sixièmement, la surveillance du contrat et le règlement des litiges reposent sur le recours à plusieurs régimes d'autorité, et notamment l'autocontrôle et l'autorité judiciaire. Compte tenu du caractère spécialisé et souvent très spécifique des prestations de recherche, la surveillance est décentralisée au niveau des exécutants du contrat qui désignent des correspondants universitaires et industriels. L'autorité est attribuée à l'un des contractants, à savoir l'entreprise, qui dispose d'une voix prépondérante, ou est partagée et exercée par consensus. Si l'autocontrôle est adapté à la situation, il peut se révéler inefficient en cas de litige (Brousseau, 1993). Le recours à l'autorité judiciaire est cependant difficile, en raison de la lourdeur de la procédure et de la complexité des objets techniques en cause, et, de fait, extrêmement rare. Les disputes sont réglées à l'amiable, entre pairs. Les chercheurs peuvent toutefois faire appel à un expert juridique extérieur, notaire ou huissier, pour faire établir l'antériorité de leurs travaux dans un domaine, ce qui peut leur être d'une grande utilité pour contracter par la suite avec un industriel.

\subsection{L'invention par les chercheurs de nouvelles règles d'appropriation des résultats : les guidelines du projet Bridge sur les lipases}

Alors que l'asymétrie de compétences juridiques entre institutions scientifiques et firmes est fréquemment soulignée (Reboul, 1995), les chercheurs sont susceptibles de jouer un rôle actif dans la création de nouvelles règles de réservation et de circulation des connaissances. 
Les chercheurs du projet de la Cee sur les enzymes lipasiques ont entrepris de rédiger des guidelines pour faciliter la coopération entre 22 participants publics et privés, dont 5 firmes largement concurrentes entre elles. Le projet fut découpé en 5 sous-projets dans lesquels les firmes se répartissaient, afin de préserver des espaces de collaboration protégés. Les guidelines régulent les échanges entre les sous-projets en définissant plusieurs degrés d'accessibilité des données en fonction du temps: "To guarantee maximum transfer of information within the entire project, a series of times periods are defined for distribution of structural and functional data obtained with the lipases studied in the project». Le droit d'accès est délimité dans l'espace, en cercles de diffusion concentriques, et dans le temps, selon des délais maximum de rétention des données : «a-team data which will be only accessible by the members of the same team. These members are bound by the confidentiality rules in their contract. Team data will be shifted to pooled data as soon as possible but not later after 6 months after their entry, whereby zero time is defined as the time of communicating a set of functional data to the members of the team, $b$ - pooled data which will be accessible through the coordinators only to all appropriate members of the 5 teams involved in the T-project. The members of the T-project will not forward pooled data to third parties without the authors/owner's agreement. Pooled data should become public data as soon as possible by means of a joint publication of all authors/owners, c- public data which will be accessible, upon acceptance of a joint publication, to the members of the Lipase Industrial Plateform, created in conjunction with this T-project, that will group all interested industries in the European Communities and EFTA countries. Industrial of the T-project agree to participate in such a plateform ".

Ces guidelines introduisent plusieurs nouveautés en matière d'appropriation et de circulation des produits de la recherche.

Elles définissent pour le propriétaire du matériel biologique un droit de priorité sur les données produites. Ce droit de réservation est temporaire, d'une durée de 3 ou de 6 mois. Cette période de réservation exclusive lui permet de tester la valeur d'usage industrielle de connaissances qui, en général, ne répondent pas immédiatement aux critères de brevetabilité. Si les données s'avèrent intéressantes, il peut envisager de déposer une demande de brevet, et si nécessaire, demander un délai supplémentaire pour faire des développements. Les guidelines instaurent un compromis original entre droit de réservation individuel et droit d'accès public. Elles permettent de prendre une avance et des protections sur des connaissances d'intérêt industriel tout en obligeant à leur divulgation rapide sous forme de publication.

Elles organisent ensuite un compromis entre protection individuelle et création collective. Si les participants bénéficient d'un droit de réservation individuel, ils sont tenus de verser leurs résultats dans le domaine collectif des vingt deux participants. Le partage des données entre les équipes accélère le développement des connaissances, dans la mesure où les connaissances relatives à une enzyme A peuvent aider à élucider une enzyme B. La constitution d'une 
collection de 25 enzymes d'intérêt industriel, rassemblée grâce à la mise à disposition temporaire d'échantillons privés, a permis d'obtenir de nouvelles connaissances sur cette famille d'enzymes grâce aux comparaisons effectuées.

Elles s'efforcent enfin de maîtriser la tension entre bien collectif, réservé aux participants au réseau, et bien public. Les guidelines définissent un accès privilégié aux données pour les participants, délimité par l'interdiction temporaire de les communiquer aux non-participants sans l'autorisation des autres membres du réseau et par l'obligation de les verser dans l'espace public dans un certain délai (de 6 ou 12 mois). Il peut en résulter des tensions autour du délai de publication, ou de la mise à disposition des résultats dans les plateformes industrielles souhaitées par la Cee pour hâter les transferts de connaissances dans le tissu industriel de la communauté. Les industriels participants au projet Lipases se sont ainsi opposés à la mise en place d'une telle plateforme destinée aux industriels non-membres.

Ces différents compromis entre droit de réservation et droit d'accès aux connaissances produisent 4 types de biens économiques : 1 - des biens privatifs, accessibles aux seuls propriétaires des données, 2- des biens collectifs, partagés entre les membres du projet pour favoriser la création collective de science. Ces biens collectifs excluent les non-membres, 3- des biens semipublics ou semi-privés, accessibles aux membres de la plateforme industrielle, 4- des biens publics accessibles à tous les utilisateurs potentiels.

L'élaboration et la mise en œuvre de ces guidelines témoignent de la créativité institutionnelle des chercheurs scientifiques. Elles ont été discutées et rédigées à l'écart des services de propriété industrielle des firmes et des organismes de recherche. Bien qu'elles n'aient pas été signées en bonne et due forme par les différentes parties, elles constituent néanmoins un engagement selon les juristes. Une autorité extérieure, administrative ou judiciaire, pourrait s'appuyer sur le document rédigé par les participants pour leur rappeler le sens de l'accord initial ou pour interpréter une requête ou une situation particulières. Elles constituent des additions au droit de la propriété industrielle pour protéger des connaissances de base et au droit contractuel communautaire pour gérer des échanges de données dans des consortia de recherche. Elles ont été écrites pour gérer des situations et des objets singuliers. Pour autant, elles ont commencé à circuler dans les projets européens de biotechnologie.

\section{2. - LES TYPES DE COMPROMIS ENTRE BIEN PRIVÉ ET BIEN PUBLIC ET LEUR IMPACT EN TERME D'EFFETS DE REPORT}

Les contrats étudiés accordent généralement la propriété exclusive des résultats à l'entreprise, ceci dans $87,9 \%$ des cas (4). Ce régime de propriété unique

(4) Ceci alors que le modèle de contrat de l'Université de Compiègne prévoit uné répartition pour moitié entre l'université et l'industriel et que le contrat-type du CNRS prévoit que "les résultats de l'étude seront la propriété du CNRS". 
est parfois renforcé par une clause qui confère à l'entreprise un monopole d'accès aux travaux du laboratoire dans le domaine du contrat : "The principal investigator undertakes and agrees not to carry out any research or other work for a commercial third party which is the same as or closely similar to that carried out under this agreement. The society acknowledges that the Principal Investigator and the Institute have an existing research contract with JouveinalSipsy on "Gastric lipases from animal sources", but the principal Investigator confirms that the objectives are non-conflicting with those of Celltech».

Seuls quatre contrats attribuent la propriété unique des résultats au laboratoire, tandis que huit établissent un régime de propriété séparée et trois un régime de propriété commune entre le laboratoire et l'entreprise. En 1981, une grande firme pharmaceutique conclut un contrat avec l'INSA de Toulouse, non pour obtenir un résultat déterminé, mais pour bénéficier d'un droit d'accès prioritaire à ses travaux. Dans ce cas, le laboratoire conserve la propriété unique des résultats (en cas de dépôt de brevet, l'entreprise bénéficiera en priorité d'une licence). Les formules de propriété séparée ou de copropriété portent sur des travaux de développement de prototypes qui ont été initiés par l'université. Ainsi, en 1980, un contrat entre l'INSA et une société fromagère attribue la propriété des résultats de l'étude à l'INSA pour tout ce qui concerne l'appareillage de fermentation et à l'entreprise pour tout ce qui touche à son milieu spécifique de fermentation. En 1991, l'UTC de Compiègne décide de collaborer avec une société d'instrumentation pour réaliser la version industrielle d'un prototype dont elle a construit plusieurs versions de laboratoire. La propriété sera répartie pour moitié. Et lorsque l'entreprise consent à partager la propriété, elle s'efforce d'obtenir un droit d'exploitation exclusif.

Pour les firmes, l'attribution de la propriété exclusive des résultats est une incitation à s'engager dans le contrat. Pour les laboratoires, une telle aliénation de leurs droits est controversée : «L'idée que l'industrie pourrait, en engageant des sommes relativement faibles dans une recherche donnée, obtenir un brevet, même si une partie du projet a été financée par des fonds publics, est évidemment une source de critiques » (Kaysen, 1986). Ces critiques sont d'autant plus vives que l'université entend capter une partie de la rente d'innovation générée par son stock de recherche et récupérer, au moins partiellement, les investissements consommés pour le développer (5).

A bien examiner les dispositions des contrats et les stratégies des laboratoires relatives à la gestion de leurs stocks de recherche, la situation est moins asymétrique qu'il n'y parait de prime abord.

(5) Une autre attitude considère que l'université doit transférer tous ses droits à l'entreprise, en contrepartie de la citation du nom des chercheurs qui ont collaboré à l'invention. Ceci pour plusieurs raisons : a- les chercheurs ne souhaitent pas consacrer de temps à la prise de brevet, b- ils s'estiment mal informés sur la propriété industrielle, c- l'université n'a aucune vocation industrielle et commerciale. Il s'établit ainsi une stricte complémentarité entre le service public de recherche, qui s`approprie les bénéfices académiques de la collaboration, et l'entreprise, qui en perçoit les bénéfices économiques. 


\subsection{Les dispositifs de protection des laboratoires universitaires}

Premier dispositif, les contrats réaffirment fréquemment, dans $45 \%$ des cas, l'antériorité des travaux et des droits des laboratoires sur les sujets traités. Le transfert de propriété ne porte que sur les résultats de la prestation réalisée en $\mathrm{t}$, le laboratoire conservant la propriété des résultats acquis en $\mathrm{t}-1$, brevetés ou non. Ce jeu sur l'antériorité des résultats du laboratoire est d'autant plus efficace que les contrats conclus s'inscrivent dans ses thèmes de recherche et qu'il détient un stock de connaissances sur le sujet. Le cas échéant, les chercheurs peuvent différer certains accords sur des sujets en cours d'exploration afin d'engranger des résultats. Le laboratoire évite ainsi le risque de se voir fermer un sujet de recherche. Il dispose ensuite d'un acquis pour négocier avec l'industriel. En cas de production d'un résultat applicable, l'accord tiendra compte de son patrimoine. Les brevets éventuels déposés par l'entreprise sont bornés par cette antériorité. D'autant que les laboratoires étudiés, à Compiègne et à Toulouse, ont pris des brevets sur leurs technologies de base dès 1968, bien avant la conclusion de contrats avec l'industrie, à partir de 1972.

Un autre mode de protection utilisé par les chercheurs de Compiègne a consisté à déposer, avant la signature d'un contrat, l'état des travaux nonpubliés du laboratoire chez un notaire. Cette pratique suffit à établir une antériorité.

Troisième procédé, les universitaires découpent l'objet du contrat de façon à limiter l'étendue des droits de l'industriel. Ceux-ci seront circonscrits à un domaine d'application spécifique : "La société demande au laboratoire de faire exécuter pendant la durée du présent contrat des études relatives à la réalisation d'électrodes utilisables pour le dosage spécifique de ce produit dans des milieux de fermentation, à l'exclusion de toutes autres applications, par exemple dans les domaines médical ou pharmaceutique " (Compiègne, 1975). Le laboratoire conserve ainsi la maîtrise des connaissances et des technologies de base, qu'il peut réutiliser par la suite dans une grande variété de projets industriels ou académiques.

Quatrième dispositif, les contrats prévoient la levée des droits de l'entreprise dès lors qu'elle n'utilise pas les résultats dans un délai donné : " Dans l'hypothèse où une application industrielle étant possible, la société déciderait de ne pas s'y intéresser, elle ne pourrait s'opposer à ce que le laboratoire au terme d'un délai de deux ans à compter de la fin de l'étude, poursuive le développement des travaux avec un autre partenaire industriel, sous réserve naturellement que les droits de la société soient préservés » (Compiègne, 1980). C'est une incitation à une utilisation effective des résultats du contrat et à leur remise en circulation rapide en cas de non-utilisation par le premier demandeur. Par exemple, des études réalisées à Compiègne en 1980 pour une société de pétrochimie ont été réutilisées dans un contrat avec une société de traitement des eaux en 1982.

Les contrats peuvent également accorder au laboratoire universitaire un droit d'utilisation des résultats à usage de la recherche : "Chaque partie pourra uti- 
liser les résultats de la collaboration pour ses propres besoins de recherche dans ses laboratoires » (Toulouse, 1987).

Enfin, afin de faire rémunérer leur apport inventif, les universitaires peuvent s'efforcer de faire inscrire dans les conventions une clause de paiement par redevance en cas d'exploitation industrielle ou commerciale d'un résultat. Cette rémunération prend parfois la forme du versement de la taxe d'apprentissage de l'entreprise au laboratoire ou du financement d'une thèse. Parfois, au contraire, le contrat spécifie qu'il n'y aura pas d'autre rémunération que forfaitaire.

\subsection{Les formules de compromis entre bien public et bien privé}

Les laboratoires prestataires de recherche sont également soumis à une obligation de secret sur les résultats des travaux qu'ils effectuent pour l'entreprise et sur les informations que celle-ci leur communique au cours de la coopération. Leur droit de publication est soumis à l'autorité de l'industriel ou à l'autorité partagée des deux partenaires réunis dans un comité de coordination qui supervise la réalisation du contrat. Ce droit de contrôle des publications peut être borné dans le temps (en général, il s'étend sur la durée du contrat plus quelques mois). Comment parvenir à concilier la priorité de publication poursuivie par l'universitaire et le droit de réservation des connaissances revendiqué par l'industriel qui veut conserver une avance sur ses concurrents et évaluer les résultats pour prendre des protections? Dasgupta et David, 1994, y voient une des principales difficultés à l'établissement des accords de coopération entre l'université et l'industrie.

En fait, les contrats étudiés correspondent rarement à l'achat d'une confidentialité totale des travaux par l'industriel. Auquel cas, le développement des connaissances du laboratoire serait entravé. Les contrats mentionnent explicitement le droit et l'objectif de publication des universitaires. Les partenaires établissent une série de compromis entre secret et publication. Premièrement, les contractants peuvent s'entendre pour séparer les informations immédiatement publiables et les informations confidentielles consignées dans un «dossier technique secret». Comment faire le tri ? Les partenaires séparent les informations attachées à la technologie spécifique de la firme ou à un milieu industriel précis, qui sont conservées dans le dossier technique secret, et les informations abstraites du contexte industriel, transposées dans un milieu de laboratoire ou reformulées dans un modèle plus général, qui peuvent être publiées. Tandis que les partenaires s'accordent pour privatiser les informations primaires d'ordre $\mathrm{n}$, les plus proches du milieu industriel, ils décident de divulguer les informations représentées dans un modèle de laboratoire d'ordre $\mathrm{n}+1$. Deuxièmement, les chercheurs peuvent organiser un domaine de recherche publique en parallèle au sujet de recherche industrielle qui leur a été confié par l'entreprise. Par exemple, choisir un matériel biologique qui est dans le domaine public pour effectuer des travaux similaires à ceux qu'ils effectuent sur la souche privée de l'industriel. Celui-ci bénéficie des résultats 
de cette recherche parallèle qui peuvent être transposés à son propre matériel biologique. Le laboratoire est libre de publier et le secret industriel est préservé. Troisièmement, les contrats peuvent s'efforcer d'accélérer la publication en fixant des bornes au droit de rétention de l'industriel (de 3, 6 ou 18 mois). Nous avons relevé quelques litiges, mais en général, les deux parties s'entendent à l'amiable pour ne pas pénaliser les étudiants en thèse. Quatrièmement, les chercheurs peuvent aussi marchander leur droit de publication en s'appuyant sur le fait que la plupart des contrats de recherche réalisés par l'université sont tarifés au coût marginal. L'entreprise ne s'acquittant pas de l'ensemble des coûts, elle ne peut prétendre à une appropriation complète des résultats. Les chercheurs industriels ajustent leurs exigences sur ce principe : ils réclament un droit de regard plus sévère dans les contrats qu'ils financent en totalité et sont plus libéraux dans les contrats abondés par une subvention. Cinquièmement, les entreprises ont enfin intérêt à accorder à leurs partenaires universitaires un droit de publier, ne serait-ce que pour recruter les laboratoires publics les plus éminents, et les soustraire ainsi à la concurrence.

Ces compromis entre publication et privatisation des connaissances sont plus ou moins favorables à l'université. Dans quelques cas, l'industriel demande un tri des informations publiables qui se révèle pénalisant pour l'universitaire. La question est très controversée dans le domaine de la structure des protéines. Le laboratoire d'ingénierie des protéines de Toulouse travaille sur des enzymes qui intéressent l'industrie pharmaceutique. Les données structurales qu'il produit ont une valeur commerciale immédiate sur le marché de la science. Il collabore avec une société spécialisée de biologie moléculaire qui est chargée de la commercialisation de ces données. L'accord passé avec cette société stipule 1- que l'université doit retenir l'information pendant la durée d'une année pour laisser à l'entreprise le temps de trouver des clients, 2- que les chercheurs sont autorisés à publier à condition que la publication ne permette pas à un tiers de retrouver les coordonnées par calcul. Il existe en effet une pratique de reverse engineering qui consiste à traiter par ordinateur les images stéréoscopiques souvent jointes à la publication pour reconstruire une structure à l'envers avec un degré de fiabilité convenable. Aussi les chercheurs ont-ils publié des schémas tronqués. Cette pratique est vivement contestée par d'autres laboratoires d'ingénierie des protéines qui sont privés de l'accès aux données complètes pour développer leurs propres recherches en comparant les différentes structures élucidées.

Ces compromis sont fonction du type de contrat passé, plus ou moins marchand. Les contrats semi-marchands sont les plus nombreux. Ils fournissent des valeurs d'usage académiques (thèses, publications) et des valeurs d'usage industrielles (résultats confidentiels, brevets). Le laboratoire n'est pas tenu à une obligation de résultat, mais de moyens et de prestation. La tarification est calculée au coût marginal. Les deux parties s'entendent sur un partage des résultats, biens privés et biens publics. Les chercheurs de l'UTC et de l'INSA de Toulouse ont créé des lieux séparés des laboratoires, des parcs scientifiques gérés par des sociétés privées de valorisation, qui ont pour vocation d'accueillir les sujets les plus confidentiels. Les contrats conclus dans ce cadre ont 
un caractère marchand nettement plus prononcé que les contrats précédents, à l'instar des prestations offertes par les sociétés privées de R\&D (l'universitaire renonce à son droit de publication, l'industriel a un droit d'appropriation très étendu, le tarif est calculé au coût complet).

\section{3. les effets de report générés par ces contrats}

Quel est l'impact de ces formules d'appropriation sur l'économie de la recherche?

Prenons un cas extrême observé à Toulouse. Une entreprise confie au laboratoire l'élaboration d'une nouvelle méthode de fermentation. Elle apporte aux universitaires des informations sur son procédé et fournit ses souches de bactéries privées. Celles-ci devront être restituées à leur propriétaire au terme du contrat. Un chercheur contractuel est recruté pour effectuer les travaux, qui sont localisés dans une pièce isolée. C'est une enclave réservée pour l'entreprise au sein de l'université afin de protéger la circulation des informations, des données et du matériel biologique. L'entreprise a capté la totalité des résultats au sortir de la pièce. Le laboratoire ne peut, ni s'en resservir en interne, ni les publier. La méthode est entièrement privatisée. La multiplication de tels contrats entraînerait une mise en sommeil des sujets universitaires dans le domaine considéré.

Le plus souvent, les contrats étudiés génèrent d'importants effets de report, sur la recherche universitaire, et indirectement, sur la recherche des contractants ultérieurs (le laboratoire jouant le rôle de redistributeur des résultats engrangés lors des contrats précédents, au moins d'une partie d'entre eux).

Les arrangements contractuels permettent au laboratoire d'incrémenter son stock de recherche avec les résultats des contrats industriels, moyennant des périodes de réservation temporaire des résultats pour l'entreprise. Une fois levée l'obligation de secret, le laboratoire a la liberté de réutiliser les données obtenues, pour développer ses connaissances ou pour produire de nouvelles applications. Ces effets de report sont facilités et accrus 1- si la durée d'aliénation des résultats est courte, 2- si l'étendue des droits de l'industriel est limitée (si les dossiers techniques secrets élaborés au terme du contrat ne recouvrent que les résultats liés au contexte industriel précis du demandeur de recherche). Le laboratoire bénéficie parfois du don des prototypes développés au cours du contrat (le centre de biotechnologie de Toulouse est peuplé de prototypes de fermenteurs construits à l'occasion de multiples collaborations industrielles, prototypes qui sont réutilisés et modifiés pour de nouvelles recherches). Il conserve également des méthodes qui lui ont été transmises par les chercheurs industriels comme des développements informatiques ou des procédés qui sont réemployés à de multiples reprises. Il conserve plus rarement les souches de matériel biologique privées apportées par l'industriel pour la réalisation du contrat (une clause prévoit généralement leur restitution au terme du contrat). Mais les chercheurs universitaires ont pu développer leurs 
recherches et publier à partir des souches privées auxquelles ils ont pu un moment accéder. Les effets de report des contrats industriels, mesurés par un paramètre $\beta$, sont fonction du degré d'appropriation des données et des résultats de recherche par le demandeur. Le paramètre $\beta$ est proche de 0 dans un cas d'appropriation complète des résultats (6). A l'inverse, il est proche de 1 dans des contrats dans lesquels l'entreprise ne demande qu'un droit de regard prioritaire avant publication, le laboratoire conservant la maîtrise des travaux et la disposition de l'ensemble des résultats.

Illustrons ce phénomène de report par deux exemples. A Compiègne, les chercheurs ont progressivement mis au point une méthode et un appareil de dosage biologique à partir d'une dizaine de contrats réalisés pour l'industrie entre 1973 et 1985. De nombreux prototypes de laboratoire ont été construits et expérimentés dans des milieux industriels variés (ils ont circulé dans des entreprises de fabrication d'instruments médicaux, de pâte à papier, de sucre, etc.). Les effets de report sont inscrits dans les différentes versions du prototype. En 1983, une société spécialisée dans la fabrication d'appareils de mesure conclut une licence de brevet pour l'industrialisation de ce procédé. Elle capte ainsi les effets de report accumulés par le laboratoire au cours de dix années de recherche contractuelle. A Toulouse, les chercheurs ont enrichi une méthode de fermentation originale en l'appliquant à une quinzaine de reprises dans des domaines industriels diversifiés qui vont de la vinification aux productions d'antibiotiques. Ce faisant, le laboratoire accumulait un stock de recherche académique disponible pour $\mathrm{n}$ utilisateurs, tout en cédant des résultats privatifs sur des applications dédiées à telle ou telle firme.

Pour l'entreprise, il n'est pas incohérent d'accepter un régime d'appropriation modérée sur les résultats d'un contrat qu'elle finance, autrement dit d'accepter qu'une partie de son effort de recherche puisse être réutilisé par le laboratoire et indirectement par des tiers (auquel cas, $B$ est non nul). C'est le sacrifice nécessaire pour bénéficier des effets de report des recherches antérieures, effectuées sur des contrats précédents ou sur des programmes de recherches initiés par l'université. Ce système relativement ouvert favorise le développement des connaissances de base et leur captation par de nombreux utilisateurs. Il est particulièrement adapté à un contexte d'accumulation et d'intégration

(6) Même dans un tel contexte, les effets de reports ne sont pas nuls, par le fait qu'ils ne consistent pas seulement à réutiliser une information i, mais à la déplacer et à la re-représenter (Latour, 1989) pour générer une information i'. Prenons l'exemple d'un contrat passé en 1983 entre une firme agroalimentaire et l'Université de Compiègne pour étudier le système enzymatique de la graine de moutarde. Deux thèses ont été soutenues, mais la circulation des résultats a été très réduite. Il n'y eu pas de publication et les deux étudiants ont été immédiatement captés par la recherche industrielle. Pour autant, ce contrat a donné l'idée aux chercheurs de l'Université de Compiègne de généraliser le système enzymatique étudié à d'autres végétaux et de construire des systèmes artificiels, par génie biologique, qui transposeraient le système naturel de la graine de moutarde à une grande variété de plantes. Les chercheurs de Compiègne ont organisé un vaste réseau technico-économique autour de ce concept. 
continue de la recherche de base, dont les résultats peuvent être utilisés pour développer une grande variété d'applications industrielles. Si bien que chaque contractant a intérêt à ne pas fermer le système, à moins que l'entreprise ait une stratégie à court terme de captation des travaux du laboratoire, sans perspective de revenir.

\section{LES TRANSFORMATIONS DE L'ÉCONOMIE DES INSTITUTIONS SCIENTIFIQUES : L'ÉMERGENCE D'«INSTITUTIONS HYBRIDES »}

Au terme de leur étude sur l'application commerciale de la technique des hybridomes, Mackenzie, Cambrosio et Keating (1988) font le constat suivant : «...there are close research ties between industry and academic institutions in the biomedical field. That observation might be further amplified by noting there is no significant distinction between the more academic institutions, such as the hospitals, and the companies, in their production of the kits. Moreover both kinds of institutions, in addition to kits, produced patents and scientific publications ». L'organisation linéaire de la science et de la technologie, confinées dans des institutions séparées, cède le pas à des interactions récurrentes entre institutions scientifiques et firmes privées, ainsi qu'à des intégrations verticales dans les différentes institutions.

\subsection{Des institutions scientifiques qui produisent pour l'académie et l'industrie}

Les laboratoires de biotechnologie et de biochimie de Compiègne et de Toulouse ont inventé dès leur origine, à la fin des années 60, des matériaux et des instruments biologiques et artificiels qui ont une double valeur d'usage, académique et industrielle (7). Ce sont des objets techniques brevetés, concédés à des industriels de l'instrumentation scientifique ou médicale, et produits à de nombreux exemplaires. Ce sont simultanément des objets d'analyse en biologie fondamentale qui permettent d'énoncer de nouvelles propriétés des systèmes biologiques. Les chercheurs sont immédiatement confrontés à la question du statut économique de leurs produits : d'un côté, ils s'engagent dans une stratégie de protection industrielle (le brevet de base sur les membranes enzymatiques date de mars 1968), en compétition avec les procédés des firmes Monsanto, Unilever et Corning Glass ; d'un autre côté, ils soutiennent des thèses et livrent des publications dans des revues internationales aussi prestigieuses que Nature. Ils sont engagés dans une double économie, de réservation des produits de l'activité de recherche, par le moyen de contrats ou de brevets, et de divulgation à l'égard de leurs pairs.

(7) Ce sont des membranes fabriquées en liant des substances biologiques (enzymes, anticorps, etc) à un support (cellulose, gélatines). Ce procédé permet de ne pas consommer l'agent biologique pendant le process industriel, et donc de pouvoir le réutiliser, et de limiter sa dénaturation dans des milieux hostiles. 


\subsection{La gestion des stocks de recherche de l'université : la tension entre privé et public}

Les laboratoires universitaires sont amenés à gérer différents degrés de confidentialité ou de publicité de leur stock de recherche. D'un côté, ils ont entrepris de gérer leur stock de recherche comme un capital, autrement dit comme un actif immobilisé qui appartient au laboratoire, protégé par le secret, des brevets ou l'avance qu'il détient, et qu'il peut céder par morceau sur le marché. L'établissement d'un marché des connaissances suppose de réserver les connaissances acquises, par exemple par la confidentialité, et de découper des prestations exclusives cédées aux clients, les plus étroites possibles. Les chercheurs peuvent retenir des informations sur un sujet dans l'attente de passer des contrats. Ils surveillent les brevets déposés par leurs concurrents, firmes ou universitaires, pour récupérer des informations sur les procédés similaires. Dans cette période de protection d'un sujet jugé " chaud », ils ne publient que les parties les plus théoriques ou diffèrent leurs publications. Et lorsqu'ils contractent avec des industriels, ils s'efforcent de découper plusieurs prestations individuelles à partir d'une technologie de base qui reste en leur possession. D'un autre côté, ils s'attachent à maintenir des connaissances et des technologies génériques libres de toute sujétion, afin de pouvoir les réutiliser et les publier librement. Les stocks de recherche des laboratoires publics sont donc composés d'éléments hétérogènes : des biens réservés en attente de commercialisation ou de publication, des biens exclusifs attribués à des entreprises sur des applications dédiées, des biens versés dans le domaine collectif ou public. On peut mettre en évidence une transformation du statut économique des éléments de ces stocks, du privé vers le public, en fonction de la durée de réservation des connaissances gardées confidentielles dans les murs du laboratoire.

\subsection{Relativiser le rôle des brevets et apprendre à les utiliser}

Le dépôt de brevets par les institutions scientifiques est très controversé (David, Mowery, Steinmueller, 1994). L'histoire des brevets déposés par les laboratoires étudiés permet 1 - d'observer un phénomène d'apprentissage des institutions scientifiques en matière de propriété industrielle, 2- d'interpréter le brevet, non seulement comme un instrument de réservation des inventions, mais aussi comme un outil de coordination entre l'université et les firmes.

A la fin des années 60, les laboratoires de biotechnologie de Compiègne et Toulouse déposent plusieurs brevets sur les inventions qu'ils mettent au point (8). L'ANVAR les y encourage fortement. Le dépôt et le suivi de ces brevets ont posé des problèmes importants aux chercheurs et aux institutions scientifiques, alors que les revenus qu'ils en ont retiré sont très faibles. Prenons

(8) Compiègne et Toulouse ont déposé au total 33 brevets sur la période 1968-1991, dont 29 entre 1968 et 1978. 
l'exemple des brevets relatifs aux membranes enzymatiques du laboratoire de Compiègne. Le brevet initial, déposé en mars 1968, complété en 1969, 1970, 1977, ne fut complètement étendu en Allemagne qu'en 1983, et aux États-Unis qu'en décembre 1990, après plusieurs années de procédures. Mal rédigé, il donnait trop d'informations sur le procédé et demandait des revendications trop larges. Il offrait ainsi de nombreuses prises à la contestation (les industriels opposants se sont appuyés sur le détail des démonstrations pour proposer des contre-exemples, ce qui allongea la procédure). Il souffrait du manque de stabilisation de l'invention, qui ne fut mieux comprise et délimitée qu'après plusieurs années de contestations et d'expérimentations. Ces brevets qui recouvrent des technologies émergentes sont vulnérables : le développement industriel peut aboutir à un produit mal protégé dans la demande initiale. Les inventeurs durent également se défendre contre leurs propres publications, considérées comme destructrices de l'activité inventive par leurs opposants. Enfin, les chercheurs de Compiègne ont pâti de leur isolement : ils n'ont pu s'appuyer sur un partenaire industriel pour défendre leur propriété.

Si le coût de dépôt et de défense de ce brevet a été assez lourd au regard des revenus monétaires engrangés, cela n'a pas été sans effets. Les chercheurs en charge du dossier se sont formés à la propriété industrielle et sont aujourd'hui mieux équipés pour décider de l'opportunité et du format d'un futur brevet. Ensuite, lors de l'établissement des contrats de recherche, le laboratoire s'est référé à son patrimoine breveté pour circonscrire les droits des industriels contractants et pour établir la dépendance des résultats qui leur étaient concédés. Les chercheurs disposaient de points d'appui pour conserver la maitrise de leur patrimoine, pour développer leurs propres sujets de recherche ou choisir leurs collaborations industrielles (9). De plus, le dépôt de brevet donne lieu à des études de faisabilité, de reproductibilité, de performances, qui n'auraient pas été réalisées autrement. Les connaissances techniques ainsi développées sont plus facilement transférables dans l'industrie. Les universités utilisent le dépôt de brevet comme un signal lancé aux acteurs industriels intéressés pour poursuivre le travail d'invention qu'elles ne peuvent mener à bien à elles seules. Les dépôts de brevet s'inscrivent dans une stratégie de recherche en coopération. Enfin, l'existence d'un brevet est une assurance demandée par certaines firmes avant de s'engager sur le sujet, particulièrement dans l'industrie pharmaceutique.

La stratégie de propriété industrielle des institutions scientifiques a sensiblement évolué depuis la fin des années 60. Elles ont renforcé leurs compétences dans le domaine, soit en recrutant des personnels à même de rédiger directement les brevets, soit en s'entourant des services réguliers de conseils en propriété intellectuelle. Leurs dépôts sont devenus plus sélectifs et leurs portefeuilles de brevets ont été triés et réduits. Leurs dépôts s'inscrivent plus clai-

(9) A contrario, les universitaires peuvent rencontrer des difficultés lorsque c'est l'industriel qui détient le brevet (cf colloque de droit sur « la propriété intellectuelle au service de la défense des droits des universités », 1995). 
rement dans des stratégies de coopération avec l'industrie : «Si la probabilité de trouver un industriel pour exploiter l'invention est égale à 0 , l'université publie » (colloque de droit, 1995). Les universitaires pensent qu'il leur sera plus facile de défendre un brevet s'il est effectivement exploité par une entreprise.

\section{CONCLUSION}

1- Les contrats de recherche, y compris les règles de bonne conduite, représentent une formule particulière d'appropriation intellectuelle dans un contexte coopératif. Jacquemin, 1987, parle « d'internalisation partielle des marchés technologiques par la coopération ». Ceci, tant par le jeu des obligations contractuelles (obligation de communiquer au bénéficiaire et de ne pas communiquer à des tiers), que par le jeu de la réciprocité qui lie les partenaires (donner, recevoir et rendre des informations, des objets, des services à un partenaire privilégié). Le caractère fermé des relations bilatérales ou des réseaux coopératifs facilite le partage des connaissances et la sécurité des transactions. Dans ce contexte, le recours au brevet n'est pas une solution privilégiée : les firmes y recourent dans seulement $22 \%$ des contrats étudiés alors que ceux-ci fournissent des prototypes ou des pilotes de laboratoire ou pré-industriels dans les deux tiers des cas.

2- Il convient de revenir sur la variété et la plasticité des outils contractuels. Les acteurs locaux disposent d'une grande liberté pour réguler les problèmes de propriété intellectuelle à l'aide des contrats (Mousseron,1995). De fait, les accords étudiés divergent quand à l'étendue et à la durée de la réservation des résultats. Or, une variation minime dans l'écriture des clauses contractuelles, par exemple la mention ou non de l'antériorité des travaux du laboratoire ou du droit de réutiliser les résultats du contrat au delà d'un certain délai, peut avoir des conséquences importantes sur le développement des connaissances universitaires. La sélection et la diffusion des bonnes pratiques contractuelles, via la circulation des modèles de contrats dans les réseaux d'universités ou via l'enrichissement des contrats-type, est une donnée importante. La formation des chercheurs est aujourd'hui facilitée par 25 années d'expériences de relations industrielles. Il s'avère que ces derniers sont à même d'élaborer des arrangements originaux qui favorisent la coopération entre académie et industrie, à l'instar des règles de bonne conduite des projets Bridge.

3- Tandis que les contrats de recherche entre l'université et l'industrie stimulent la création de nouvelles connaissances, ils définissent généralement un régime de compromis entre biens publics et biens privés, avec une grande variété des arrangements possibles (en jouant sur la durée et l'étendue du droit de réservation et sur la sélection des connaissances et des données concernées). Les règles de bonne conduite du projet Lipases introduisent une nouveauté, celle de bien collectif, qui repose sur le partage des données entre les participants d'un consortium de recherche afin de stimuler leur création commune. Les contrats sont des outils de gestion concertée des externalités posi- 
tives de la science, via le droit de réutilisation des résultats ou leur publication. Ils donnent lieu à des externalités locales, via un réseau d'échange délimité par le contrat, ou générales, via le droit à la publication des résultats. On s'aperçoit que les firmes engagées dans une collaboration durable avec l'université peuvent avoir intérêt à participer à un système relativement ouvert. D'une part, pour recruter les laboratoires les plus réputés qui exigent de pouvoir publier. D'autre part, pour bénéficier des effets de report des précédents contrats réalisés par le laboratoire, qu'elles n'ont pas financé, mais qui leur reviennent en partie.

4- L'économie des institutions scientifiques est en train de changer (Etzkowitz et Peters, 1991). Elles gèrent des situations et des produits hybrides : d'un côté, elles déposent des brevets et concèdent des licences, de l'autre, elles publient leurs résultats dans des revues internationales (ce qui les conduit à gérer de manière attentive le secret industriel, les dépôts de brevets et les délais de publication). Elles ont organisé depuis le début des année 70 un véritable marché de la science et de la technologie autour des universités (associations de recherche sous contrat, sociétés de transfert, sociétés privées de R\&D). Bien qu'elles soient majoritairement financées par des fonds publics, elles collectent des fonds privés qui ont un caractère incitatif sur leurs travaux et qui exercent un effet de levier sur leurs moyens. Le cas échéant, elles organisent le recyclage des bénéfices tirés des contrats industriels dans l'équipement et le fonctionnement de la recherche publique. Des parcs scientifiques hébergent sur le sol de l'état des laboratoires industriels qui jouissent de contrats d'occupation à long terme, des sociétés privées de valorisation dans lesquelles l'établissement public participe majoritairement aux côtés de firmes industrielles, des laboratoires mixtes. Le modèle d'organisation de la recherche qui se dessine repose sur la coordination, et localement sur l'intégration dans les différentes institutions, des domaines public et privé, plutôt que sur leur séparation. Les situations observées ne relèvent pas non plus d'un phénomène de privatisation unilatérale des institutions scientifiques (Webster, 1989). La maîtrise des coordinations à établir entre le domaine public et le domaine privé de la science demande des apprentissages de la part des universités, notamment en matière de propriété intellectuelle, et l'invention de nouveaux droits d'accès aux connaissances qui rendent compatibles un certain degré de protection individuelle, l'invention collective et la diffusion des connaissances dans l'espace public. 


\section{BIBLIOGRAPHIE}

ARROW K.J. (1962), "Economic welfare and the allocation of resources for invention ", reprinted in collected papers of K.J. ARROW, volume 5, Production and capital, Harvard University Press, 29-35.

BROUSSEAU E. (1993), L'économie des contrats, Paris, Puf, 368 p.

CALLON M. (1992), « Sociologie des Sciences et Économie du changement technique : l'irrésistible montée des réseaux technico-économiques ", Ces réseaux que la raison ignore, Paris, L'Harmattan, 246 p.

CASSIER M. (1995), Les contrats de recherche entre l'université et l'industrie: l'émergence d'une nouvelle forme d'organisation industrielle, thèse de socio-économie de l'École des Mines, Paris, 605 p.

COHEN W., FLORIDA R., GOE W.R. (1994) "University-Industry Research Centers in the United States ", Carnegie Mellon University, 38 p.

COHENDET P., HERAUD J.A., ZUSCOVITCH E. (1992), "Apprentissage technologique, réseaux économiques et appropriabilité des innovations ", Technologie et richesses des nations, Paris, Économisa, 63-78.

DASGUPTA P. and DAVID P. (1994), «Toward a new economics of science », Research Policy, $23,487-521$.

DAVID P., MOWERY, D.C., STEINMUELLER W.E. (1994), "University-industry research collaborations : managing missions in conflict". Conference on "University goals, Institutionnal Mechanisms and the 'industrial Transferability' of research ", Stanford, 23 p.

ETZKOWITZ H. and PETERS L.S. (1991), «Profiting from knowledge : organisational innovations and the evolution of academic norms ", Minerva, volume XXIX, $n^{\circ} 2$, summer, 133 166.

GAFFARD J.L. (1989), "Marchés et organisation dans les stratégies technologiques des firmes industrielles », Revue d'Économie Industrielle, $\mathrm{n}^{\circ}$ 48, 2ème trimestre 89, 35-51.

GLUCK M.E., BLUMENTAL D., STOTO M. (1987), « University-industry relationships in the life sciences : Implications for students and post-doctoral fellows ", Research Policy, 16, 327-336.

JACQUEMIN A. (1987), "Comportements collusifs et accords en recherche-développement ». Revue d'Économie Politique, 97 (1), janv-févr., 1-23.

KAYSEN C. (1986), "Science, gouvernement et industrie aux États-unis : quoi de neuf ?", Les enjeux du changement technologique, Paris, CPE Économica, 133-150.

LATOUR B. (1989), La science en action, Paris, La Découverte, 450 p.

LIVET P. et THÉVENOT L. (1991), "L'action collective ". Colloque sur l'Économie des conventions. École Polytechnique. Paris. mars, 30 p.

MACKENZIE M.. CAMBROSIO A.. KEATING P. (1988). "The commercial application of a scientific discovery: the case of the hybridoma technique ". Research Policr. 17. 155-170. 
MOUSSERON J.M. (1995), "L'exploitation des créations émanant de l'université », Colloque sur "La propriété intellectuelle au service de la défense des droits des universités ", 59-67.

NELSON R.R., ROMER P.M. (1994), « Science, Economic Growth, and Public Policy », 4974.

PERROT A. (1992), «Asymétries d'information, qualité des produits et contrats », Revue Gérer et Comprendre, mars, 18-22.

PISANO G. P. (1991), «The governance of innovation: Vertical integration and collaborative Arrangements in the biotechnology industry ", Research Policy, 20, 237-249.

REBOUL Y. (1978), Les contrats de recherche, Paris, Librairie technique, 306 p.

REBOUL Y. (1995), "Les contrats de recherche ", Colloque sur "La propriété intellectuelle au service de la défense des droits des universités ", 43-59.

WEBSTER A., 1989, «Privatization of public sector research : the case of a plant breeding institute ", Science and Public Policy, 16 (4), 224-232.

WILLIAMSON O. (1981), «The modern Corporation : origins, evolution, attributes », Journal of Economic Literature, Vol XIX, december, 1537-1568.

\section{ANNEXE}

TABLEAU 1: Répartition de la propriété des résultats

\begin{tabular}{|c|c|c|c|c|c|}
\hline $\begin{array}{l}\text { Propriété unique } \\
\text { de l'entreprise }\end{array}$ & $\begin{array}{l}\text { Propriété unique } \\
\text { du laboratoire }\end{array}$ & $\begin{array}{l}\text { Propriété séparée } \\
\text { de l'entreprise } \\
\text { et du laboratoire }\end{array}$ & $\begin{array}{l}\text { Copropriété } \\
\text { de l'entreprise } \\
\text { et du laboratoire }\end{array}$ & $\begin{array}{l}\text { Propriété attribuée } \\
\text { au financeur public } \\
\text { (ANVAR) }\end{array}$ & $\begin{array}{l}\text { Attribution } \\
\text { à définir } \\
\text { par un avenant }\end{array}$ \\
\hline 139 & 4 & 8 & 3 & 2 & 2 \\
\hline
\end{tabular}

TABLEAU 2 : Les brevets déposés par les laboratoires et par les entreprises contractantes

\begin{tabular}{|l|l|l|l|l|}
\hline & Compiègne & Toulouse & Marseille & Total \\
\hline Brevets déposés par l'unisersité & 16 & 17 & 1 & 34 \\
\hline Brevets déposés par la firmé contractante & 3 & 21 & 5 & 29 \\
\hline
\end{tabular}

